\title{
INJECTION-LOCKING IN FABRY-PEROT QUANTUM-WELL LASERS
}

\author{
X. Jin and S. L. Chuang
}

Optoelectronic devices and fiber optic communication have revolutionized the Internet, longdistance phone calls, and cable TV for the past few years. The massive amounts of data transmission require large amounts of bandwidth, achievable through high-speed modulation of the laser sources. In this study, we report experiment results and theoretical calculations of dc and small-signal modulation of a side-mode injection-locked Fabry-Perot (FP) laser.

We first measure the lasing spectra of the master laser and the injection-locked quantum-well test laser. The master laser is a tunable laser source emitting at $1557.45 \mathrm{~nm}$, which is close to the fifth side-mode on the longer wavelength side of the lasing mode of the test laser. In Fig. 1, the dashed line shows the laser emission spectrum of the test laser with the main peak wavelength at $1551.2 \mathrm{~nm}$. This is a typical FP laser spectrum, and the side-mode suppression ratio (SMSR) of the laser is around $20 \mathrm{~dB}$. When the external master laser light is injected into the test laser biased above threshold, the injected light competes with the spontaneous emission of the laser for amplification. When the external light is strong enough and close to the eigen-frequency of the test laser, it is amplified since there is gain available. At the same time it saturates the gain of the other modes and extinguishes all the other free-running modes. Once a perfect locking state is reached, all of the power of the test laser is emitted at the optical frequency of the master laser, as shown by the solid line in Fig. 1. Injection locking greatly improves the SMSR of the test laser and shifts the lasing wavelength from $1551.2 \mathrm{~nm}$ to $1557.45 \mathrm{~nm}$. This side-mode injection locking of a FP laser has the advantage of mode selection over that of a single-mode DFB laser. This intermodal injection locking can switch the information from the free-running mode to any side mode as long as the injection-locking condition is satisfied, and can be used for optical wavelength conversion in wavelength division multiplexing (WDM) channel selection [1][2].

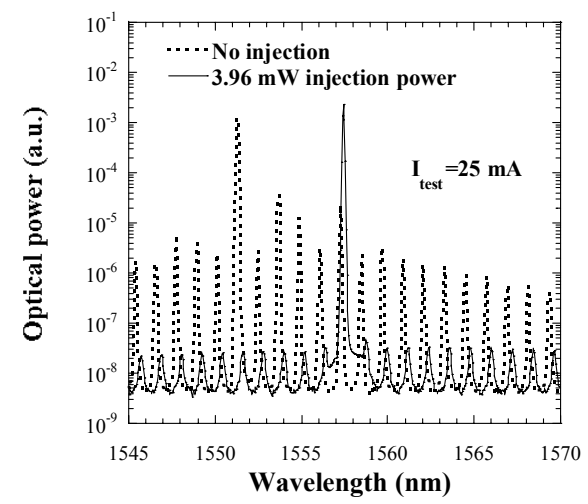

Fig. 1 Measured optical spectrum of the test laser without injection (dashed line) and with injection (solid line) at $25 \mathrm{~mA}$ bias.

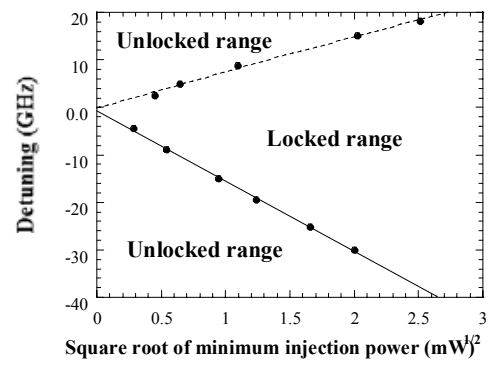

Fig. 2 Locking range dependence on both injected power and detuning for a fixed test laser bias of $30 \mathrm{~mA}$ at $25^{\circ} \mathrm{C}$. 
We also measure the overall locking range of a FP semiconductor laser, which is determined by both frequency detuning and the external injection power at a fixed test laser bias, shown in Fig. 2. By fitting the data, we obtain the linewidth enhancement factor of the test laser is 1.8, which is in agreement with a measurement using an independent method based on amplified spontaneous emission spectroscopy [3]. We will use this value in our theoretical calculation of the modulation response.

The small-signal amplitude-modulation response of the injection-locked test signal is measured when a dc master laser signal is injected into the test laser biased above the threshold with small-signal modulation. Fig. 3 shows the modulation response of the injection-locked test laser under a constant test laser bias $\mathrm{I}=30 \mathrm{~mA}$ at different injection powers. We show the improvement of $3 \mathrm{~dB}$ modulation bandwidth of an injection-locked FP laser, which is twice of its free-running value. The relaxation frequency is 3.5 times of its free-running value. The injected signal reduces more unwanted fluctuations and feedback, more stimulated emission than random spontaneous emission occurs, and enhances the peaks. For injection locking laser system, the photon density of the slave laser is coupled in phase, which enhances the bandwidth. Our theoretical model also requires setup coupling between the photon density and its phase terms. This important characteristic of injection-locked semiconductor lasers is called amplitude and phase coupling. We also point out that the small-signal modulation of injection-locked lasers still suffers low frequency roll-off, which comes from the carrier transport effect and parasitic effect of the bias circuit. This phenomenon is also shown in the data of distributed feedback (DFB) laser provided by Ref. [4]. Finally, we improve the existing small-signal model for injection locking by adding the optical confinement factor of separate-confinement-heterostructure (SCH) QW lasers, nonlinear gain saturation of the slave laser due to the master laser, and low frequency roll-off due to carrier transport and parasitic effects. Our model includes all relevant phenomena, either observed experimentally or predicted theoretically and shows good agreement with our experimental results.

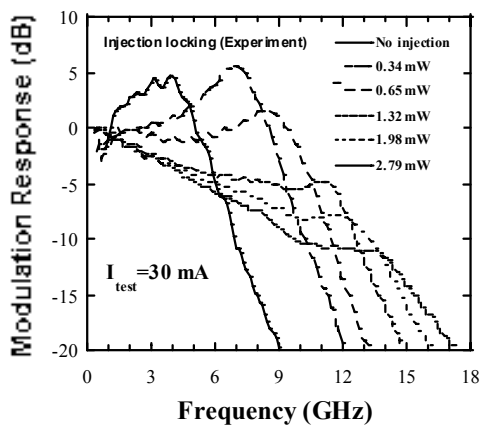

(a)

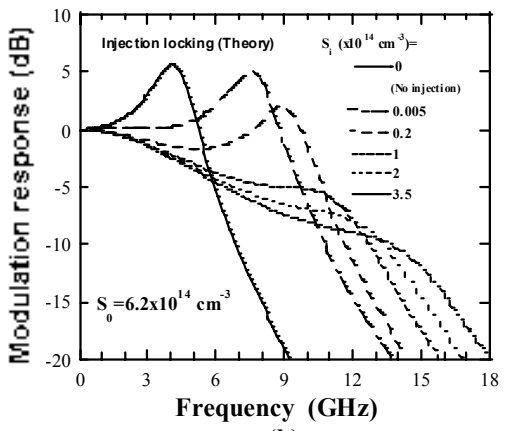

(b)

Fig. 3 Experiment data (a) Theoretical calculation (b) of the small-signal modulation response of an injection-locked test laser.

\section{REFERENCES}

[1] Y. Hong and K. A. Shore, "Locking characteristics of a side-mode injected semiconductor laser," IEEE J. Quantum Electron., vol. 35, pp. 1713-1717, 1999.

[2] J. Horer and E. Patzak, "Large-signal analysis of all-optical wavelength conversion using two-mode injection-locking in semiconductor lasers," IEEE J. Quantum Electron., vol. 33, pp. 596-608, 1997.

[3] T. Keating, S. H. Park, J. Minch, and S. L. Chuang, "Optimal refractive index changes for cross-gain and cross-phase modulation," Proc. SPIE, vol. 3283, pp. 314-322, 1998.

[4] X. J. Meng, T. Chau, and M. C. Wu, "Experimental demonstration of modulation bandwidth enhancement in distributed feedback lasers with external light injection," Electron. Lett., vol. 34, pp. 2031-2032, 1998. 